\title{
Vorwort zur zweiten Auflage
}

Zwischen dem Erscheinen der ersten und der Vorbereitung der zweiten Auflage der vorliegenden Arbeit liegt eine Zeitspanne von nur etwas mehr als einem halben Jahr. Der Wunsch des Verlages, das sich an der hier behandelten Thematik zeigende rege Interesse ohne große Verzögerung durch eine Neuauflage weiter zu befriedigen, war für den Autor AnlaB, diesem Wunsch kurzfristig zu entsprechen.

Die Zuspitzung der ökonomischen und sozialen Widersprüche in der BRD, wie auch im imperialistischen Lager insgesamt, haben die bisher getroffenen Einschätzungen über den Neokeynesianismus im Grunde nur bestätigt: der Neokeynesianismus als staatsmonopolistische Regulierungstheorie erweist sich außerstande, die Widersprüche des heutigen Kapitalismus zu lösen. Diese monopolbourgeoise wirtschaftstheoretische Doktrin bildet jedoch nach wie vor, auch trotz ihrer zutage tretenden Schwächen und trotz kritischer Einwände aus Kreisen der bürgerlichen Ökonomen, die vorherrschende politökonomisch-theoretische Konzeption der Monopolbourgeoisie im Bereich der staatsmonopolistischen Regulierungstheorien. Auf der 9. Tagung des ZK der SED, die Ende Mai 1973 stattfand, wurden u. a. auch die grundlegenden Entwicklungsprozesse des heutigen Kapitalismus eingeschätzt. In diesem Zusammenhang wurde hervorgehoben, daß „die Verschärfung der allgemeinen Krise des Kapitalismus unübersehbar ist“, daß „der gegenwärtige Kapitalismus ein Bild (bietet), das durch eine Häufung von Widersprüchen und Krisenerscheinungen gekennzeichnet ist", und daß sich selbst im Urteil der monopolbourgeoisen Wortführer der heutige Kapitalismus als „kranke Gesellschaft" repräsentiert.1 Diese grundlegenden Charakteristika des zeitgenössischen Kapitalismus stellen an die bürger-

1 Vgl. Erich Honecker, Zügig voran bei der weiteren Verwirklichung der Beschlüsse des VIII. Parteitages der SED, Berlin 1973, S. 23 ff. 
liche politische Ökonomie in ihrer Gesamtheit erhöhte Anforderungen auch hinsichtlich der Effektivität des staatsmonopolistischen Regulierungsinstrumentariums, aber auch hinsichtlich ihrer ideologisch-apologetischen Funktion. Das gilt uneingeschränkt auch in bezug auf den Neokeynesianismus. Mehrere Aspekte dürften dabei von genereller Bedeutung sein für die weitere Entwicklung dieser politökonomischen Doktrin: die beschleunigte Internationalisierung des Monopolkapitals, insbesondere im Rahmen der EWG, das rasche Voranschreiten der Konzentration und Zentralisation des Kapitals und die damit einhergehende wachsende Rolle der imperialistischen Unternehmerverbände, die enorm gesteigerte Ausbeutung einschließlich der Verschärfung des ökonomischen Klassenkampfes sowie nicht zuletzt die permanenten Währungskrisen der letzten Zeit und die galoppierende Inflation, die zu einer chronischen Krankheit des Kapitalismus geworden ist.

Es versteht sich fast von selbst, daß diese Entwicklungstendenzen im gegenwärtigen Kapitalismus die weitere Gestaltung des Neokeynesianismus beeinflussen werden. In dem Maße beispielsweise, wie die Rolle der imperialistischen Unternehmerverbände auch als Institution zur Regulierung des gesellschaftlichen Reproduktionsprozesses zunimmt, wird für sie die keynesianische Wirtschaftstheorie von gesteigertem Interesse werden, wobei die Neokeynesianer ihrerseits den konkreten Regulierungsbedürfnissen von Gruppeninteressenvertretern werden Rechnung zu tragen haben. Oder aber: Mit der Internationalisierung des Monopolkapitals wird sich zweifellos der Einfluß der keynesianischen Wirtschaftstheorie weiter vertiefen. Die sich im internationalen Rahmen komplizierter gestaltenden Reproduktionsbeziehungen zwingen die Monopólbourgeoisie dazu, den ökonomisch-theoretischen Aspekten dieser Entwicklung, und sie werden.bekanntlich durch die Neokeynesianer abgedeckt, wachsende Aufmerksamkeit zu widmen. Die Zuspitzung des Klassenkampfes wiederum wird die ideologischen Aktivitäten der Monopolbourgeoisie intensivieren. Dabei wird man sich künftighin auch auf die keynesianischen Dogmen stützen müssen.

In der vorliegenden zweiten Auflage konnte auf die hier skizzierten Probleme noch nicht detaillierter eingegangen werden, da deren Entfaltung selbst erst noch näher zu beobachten ist. Jedoch war es notwendig, neben einer Reihe von Aktualisierungen, der in jüngster Vergangenheit sich entfaltenden Diskussion in bürgerlichen Kreisen um die Attraktivität der keynesianischen Wirtschaftslehre für die Interessen der Monopolbourgeoisie im Zusammenhang mit den geldmengentheoretischen Regulierungsvorschlägen der Anhänger des amerikanischen Ökonomen Milton Friedman breiteren Raum zu widmen. Diese zusätzlichen kritischen Über- 
legungen wurden dort eingefügt, wo sie ihrem Gegenstand entsprechend einzuordnen sind: im Abschnitt „Grenzen der staatsmonopolistischen Wirtschaftsregulierung" des 6. Kapitels.

Der Autor wünscht sich für die zweite Auflage seines „Neokeynesianismus“ eine ebenso schnelle Verbreitung, wie sie bei der vorhergehenden gegeben war, denn der letzte $Z_{\text {weck }}$ dieser Arbeit besteht nach wie vor darin, einen Beitrag zur ideologischen Auseinandersetzung mit der modernen bürgerlichen politischen Ökonomie zu leisten.

Kritischen Hinweisen, Anregungen und Vorschlägen zur Verbesserung der vorliegenden Schrift sieht der Autor mit Interesse und Dankbarkeit entgegen.

Berlin, im Juni 1973

Klaus Müller 
